\title{
Ureteric obstruction due to fungus-ball in a chronically immunosuppressed patient
}

\author{
Niall F. Davis, MD; Lisa G. Smyth, MD; Elizabeth Mulcahy, MD; Tim Scanlon, MD; Liam Casserly, MD; \\ Hugh D. Flood, MD
}

Department of Urology, Mid-Western Regional Hospital, Dooradoyle, Limerick, Ireland

Cite as: Can Urol Assoc J 2013;7(5-6):e355-8. http://dx.doi.org/10.5489/cuaj.1214 Published online May 13, 2013.

\section{Abstract}

Candida albicans is a fungus that can cause opportunistic urinary tract infections in immunocompromised patients. Disseminated fungaemia secondary to Candida albicans is associated with considerable mortality and therefore merits aggressive treatment. Diagnostic investigations for urosepsis and disseminated fungaemaia secondary to Candida albicans include positive urine and blood cultures. Herein, we describe an extremely unusual case of disseminated fungaemia associated with an obstructive fungusball in the distal ureter of an immunosuppressed patient. We also describe a novel application of an established endourological technique for managing this clinical scenario and discuss appropriate perioperative management strategies.

\section{Introduction}

Candida albicans is a diploid fungus that can cause opportunistic infections in immunocompromised patients. Within the general population, it is a relatively rare causative pathogen in urosepsis. ${ }^{1}$ Fungaemia secondary to Candida albicans is associated with high morbidity and mortality rates due to the fungi's polyphenic nature and ability to form virulent hyphae in patients that are immunosupressed. ${ }^{2}$ Herein, we report an extremely unusual case of disseminated fungaemia secondary to a fungus-ball causing distal ureteric obstruction in a chronically immunosupressed patient. To the best of our knowledge, there are no previously published case reports describing an acute distal ureteric obstruction secondary to the accumulation of fungi. We also describe a novel application of an established endourological technique that relieved the fungal obstruction and efficiently removed the fungusball from the infected genitourinary tract.

\section{Case report}

An 80-year-old female presented to the emergency department complaining of pyrexia, rigors and left-sided flank pain for the preceding 36 hours. Her relevant medical history included polymyalgia rheumatica and rheumatoid arthritis, for which she was on long-term immunosuppressant therapy. Blood cultures were positive for candida albicans and urine microscopy was positive for candida spores. A computed tomography of the kidney, ureter and bladder (CT KUB) demonstrated left-sided hydronephrosis (Fig. 1, part a) and a dilated ureter down to the region of the left uretero-vesical junction where there was a small exophytic bladder mass (Fig. 1, part b). Notably, no calculus was seen and a provisional diagnosis of acute fungaemia secondary to a distal left ureteric mass was made. A percutaneous leftsided nephrostomy was inserted to relieve the obstruction; however a follow-up nephrostogram (Fig. 2, part a) showed a persistent linear filling defect in the distal left ureter with obstruction to the flow of contrast into the bladder (Fig. 2, part b). A subsequent flexible cystoscopy was performed and fungating white masses were observed protruding from a swollen left intravesical ureter at multiple points. Histopathology of a biopsy of the fungus-ball confirmed the presence of fungal hyphae (Fig. 3).

Subsequently, the patient underwent a rigid cystoscopy where multiple fungal masses were observed perforating the left ureter proximal to the ureteric orifice (Fig. 4, parts $a, b)$. Transurethral resection of the left ureteric orifice and distal intravesical ureter were performed and all traces of fungus removed leaving a widely patent ureteric opening proximally (Fig. 4, part c). Rigid ureteroscopy confirmed the presence of a clear left ureter (Fig. 4, part d). Postoperatively, a renal ultrasound was performed which showed no recurrence of the hydronephrosis and no evidence of distal ureteric dilatation. The percutaneous nephrostomy was clamped and later removed. She was discharged after completing a 2-week course of intravenous antifungal therapy (caspofungin $50 \mathrm{mg}$, once daily) and remains well. Check cystoscopy 


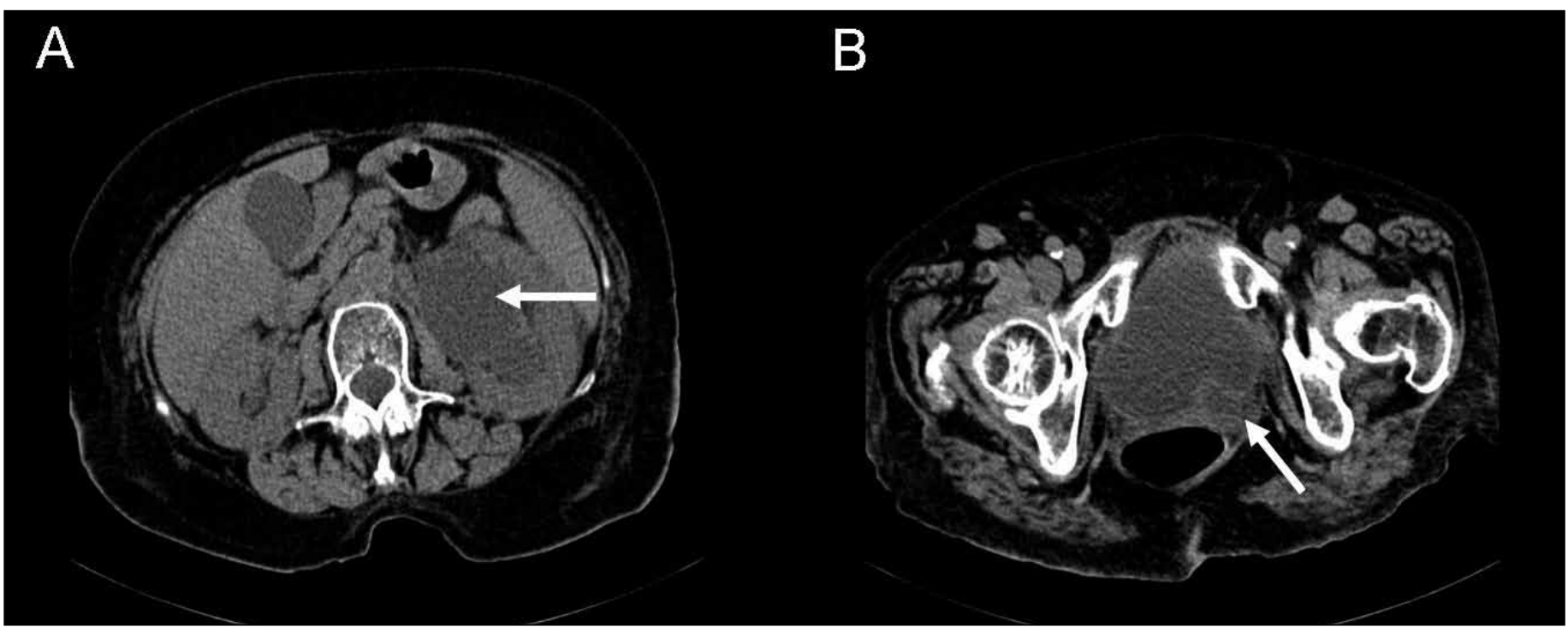

Fig. 1. A computed tomography kidney, ureter, bladder (CT KUB) demonstrating left-sided hydronephrosis (A, arrow) and a small exophytic bladder mass adjacent to the left uretero-vesical junction ( $\mathrm{B}$, arrow).

at 1 month showed a well-healed, widely-patent left ureteric orifice.

\section{Discussion}

It is widely accepted that advances in medical technology, cancer therapy, organ transplantation and chemotherapeutic agents have reduced the mortality rates of numerous life-threatening diseases. However, it is also apparent that a multitude of patients benefiting from these significant advances are also at risk for opportunistic fungal infections. The Candida species of fungi are the most common cause of infections in immunocompromised patients. In this report, we describe an unusual case of Candida albicans causing an acute uretero-vesical obstruction and disseminated fungaemia in a chronically immunosuppressed patient. Although there are documented cases of fungi (secondary to Candida albicans) occurring in renal pelves, fungal accumulation in the region of the distal ureter remains unique to this report. Another interesting and unique feature is that the obstructing fungal mass appeared to have gradually perforated through the distal left ureter into the bladder (Fig. 4, part b).

Previously, reports of fungal urosepsis have identified long-term antibiotic therapy, immunocompromising states

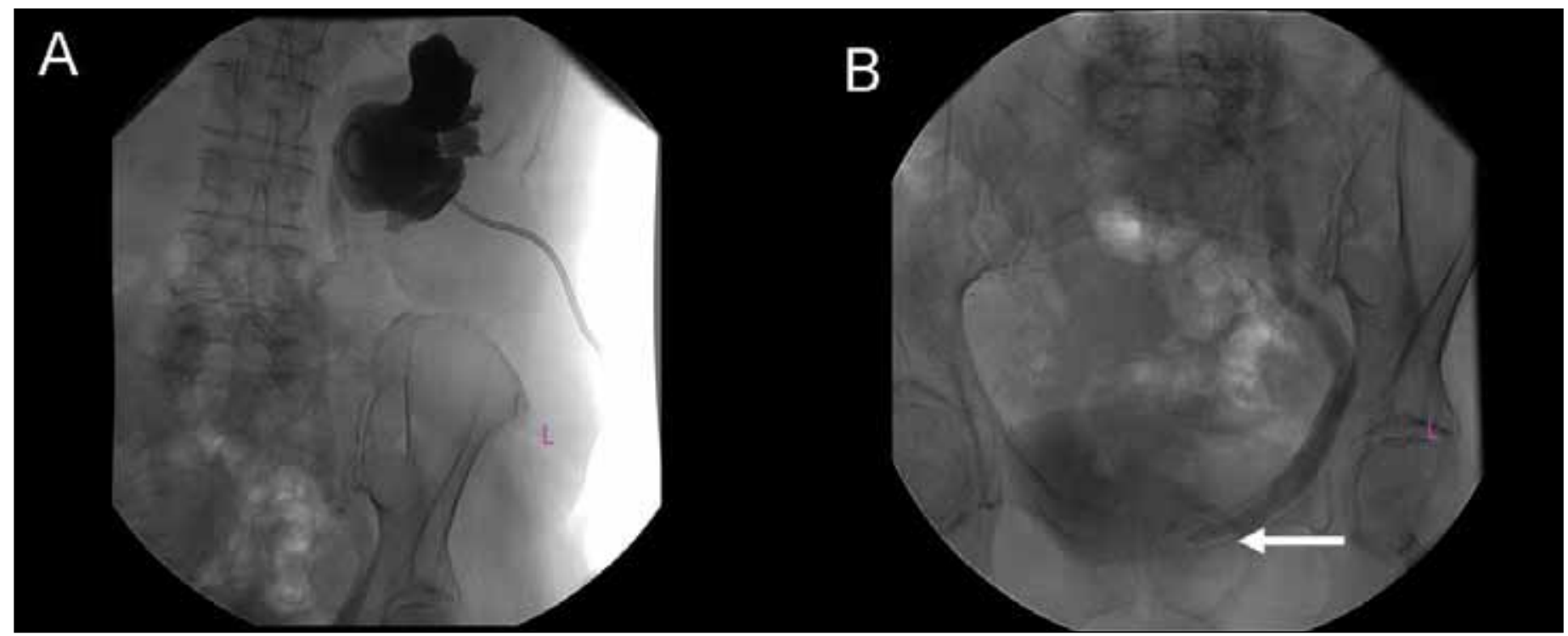

Fig. 2. A follow-up nephrostogram (A) was performed to assess whether the distal ureteric obstruction had resolved after inserting a percutaneous nephrostomy tube. The nephrostogram demonstrated persistent persistent obstruction of the distal left ureter with very poor flow of contrast into the bladder (B, arrow). 


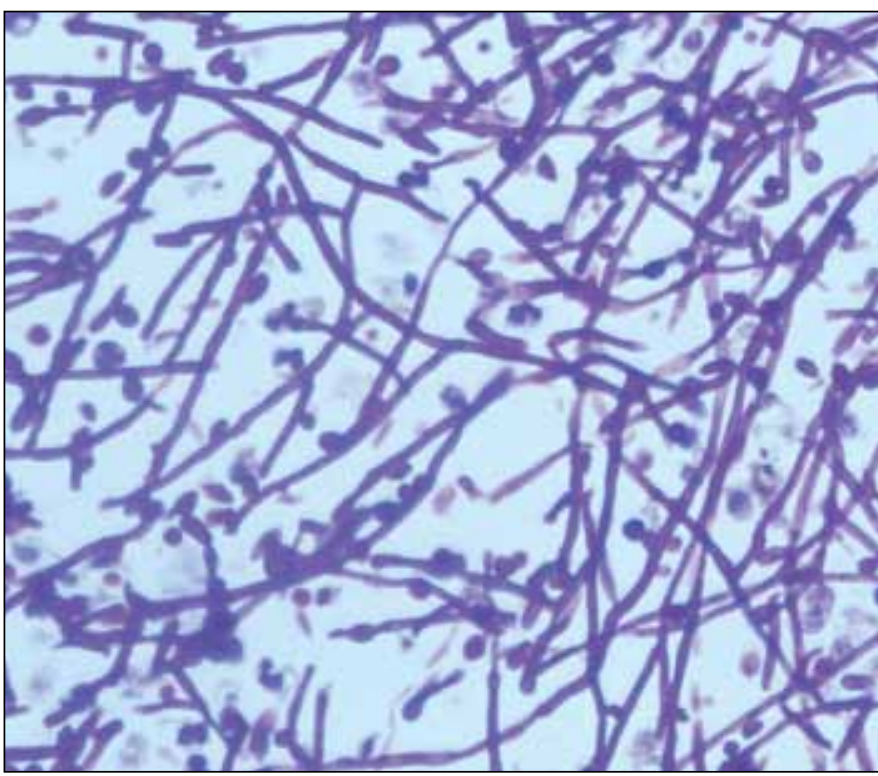

Fig. 3. Candida spores and hyphae, Pas (fungus) $\times 60$.

(e.g., diabetes mellitus, chemotherapy) and prolonged indwelling ureteral stents as important risk factors for disseminated fungaemia..$^{3-4}$ Our patient's relevant risk factors are also consistent with these studies as she had been on long-term immunosuppressant therapy for rheumatoid arthritis and polymaylagia rheumatica. These predisposing factors are important as mortality rates associated with urosepsis and disseminated fungaemia have remained high at $30 \%$ to $40 \%$ for the last number of years. ${ }^{5}$ Unfortunately, appropriate management strategies are often hampered by delays in diagnosis and by a paucity of reliable diagnostic modalities. As a result disseminated candidiasis causes more fatalities than any other systemic mycosis. ${ }^{6}$ It is also notable that blood cultures taken from our patient during her presentation were positive for the Candida albicans species because other studies have demonstrated that the sensitivity of blood cultures is relatively low at $50 \%$ to $60 \% .{ }^{4}$ Importantly, positive urine cultures and blood cultures almost always imply the presence of disseminated genitourinary fungaemia. Therefore, prompt and aggressive treatment should be encouraged by healthcare professionals.

After conducting a literature search on "urosepsis" and "disseminated fungaemia," we found 17 published case reports describing accumulated fungus-balls in the genitourinary tract. However, in these studies the accumulated fungus has been localized to the renal pelvis and/or pelviureteric junction. ${ }^{8}$ In most cases, resolution occurs after inserting a percutaneous nephrostomy tube and administering concomitant intravenous antifungal therapy. ${ }^{8}$ Unfortunately, effective retrograde fungal drainage was precluded in this case (as evident by the persistent obstruction in her followup nephrostogram) due to the unusual distal location of

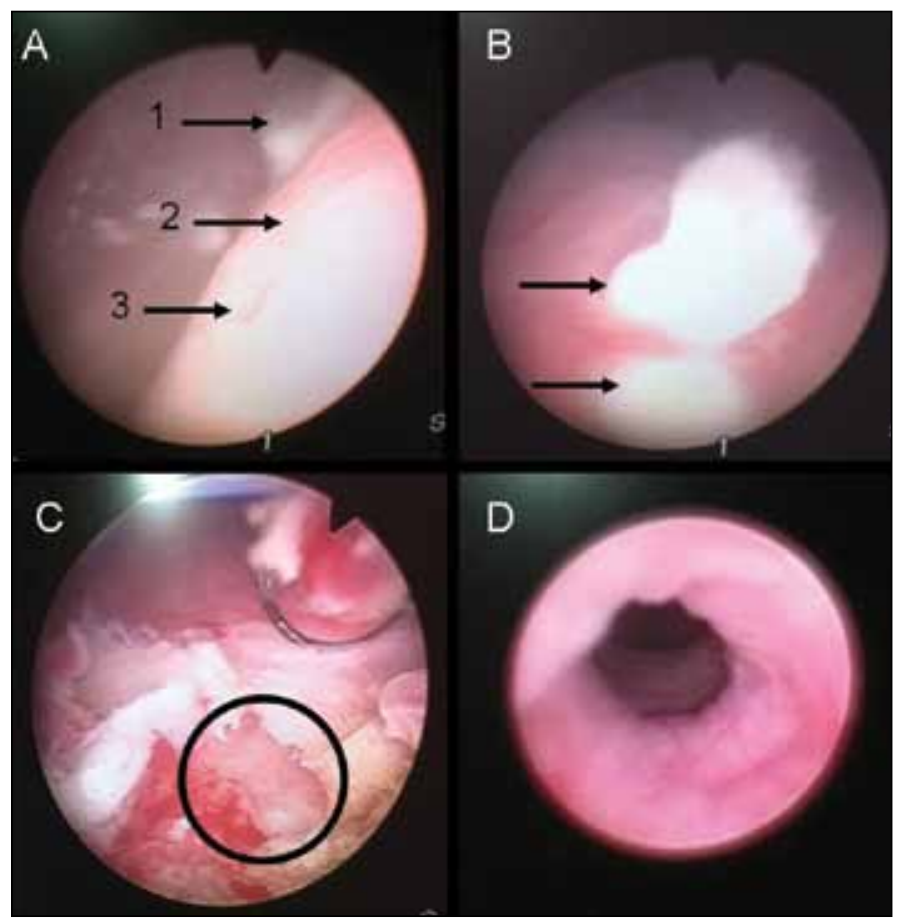

Fig. 4. Intraoperative images illustrating transurethral resection of the left ureteric orifice. At cystocopy fungal accumulation ( $A$, arrow 1$)$ and a swollen left intravesical ureter $(A$, arrow 2$)$ adjacent to a normal appearing ureteric orifice was observed ( $A$, arrow 3 ). On closer inspection multiple fungating white masses were observed perforating from the swollen left intravesical ureter at multiple points ( $B$, arrows). A transurethral resection of the left ureteric orifice was then performed and all fungal masses removed leaving a widely patent ureteric opening ( $C$, circle). Afterwards, rigid ureteroscopy confirmed the presence of a patent left ureter (D).

the accumulated fungus. After considering different surgical approaches, a transurethral resection of the left ureteric orifice was selected due to its relative simplicity and minimallyinvasive nature. Encouragingly, the early and later clinical outcomes were excellent with this combined medical and surgical management.

\section{Conclusion}

We report the first case of urosepsis and disseminated fungaemia secondary to the accumulation of a distal ureteric fungal-ball. The major risk factor in our patient was chronic immunsosuppression therapy. We also describe a novel application of an established, minimally invasive, endourological technique that relieved the ureteric obstruction by removing the fungus-ball impacted in the distal ureter.

Competing interests: None declared.

This paper has been peer-reviewed. 
Davis et al.

\section{References}

1. Bjerklund Johansen TE, Cek M, Naber K, et al. Prevalence of hospital-acquired urinary tract infections in urology departments. Eur Urol 2007;51:1100-11; discussion 1112. http://dx.doi.org/10.1016/j. eururo.2006.08.012

2. Berman J, Sudbery PE. Candida Albicans: a molecular revolution built on lessons from budding yeast. Nat Rev Genet 2002;3:918-30. http://dx.doi.org/10.1038/nrg948

3. Beck SM, Finley DS, Deane LA. Fungal urosepsis after ureteroscopy in cirrhotic patients: a word of caution. Urology 2008;72:291-3. http://dx.doi.org/10.1016/i.urology.2008.01.005

4. Pappas PG, Rex JH, Lee J, et al. A prospective observational study of candidemia: epidemiology, therapy, and influences on mortality in hospitalized adult and pediatric patients. Clin Infect Dis 2003;1;37:634-43.
5. Morgan J. Global trends in candidemia: review of reports from 1995-2005. Curr Infect Dis Rep 2005;7:429-39. http://dx.doi.org/10.1007/s1 1908-005-0044-7

6. Holzheimer RG, Dralle H. Management of mycoses in surgical patients - review of the literature. Eur J Med Res 2002;7:200-26.

7. Malani AN, Kauffman CA. Candida urinary tract infections: treatment options. Expert Rev Anti Infect Ther 2007;5:277-84. http://dx.doi.org/10.1586/14787210.5.2.277

8. Sadegi BJ, Patel BK, Wilbur AC, et al. Primary renal candidiasis: importance of imaging and clinical history in diagnosis and management. J Ultrasound Med 2009;28:507-14.

Correspondence: Mr. Niall F. Davis, Department of Urology, Mid-Western Regional Hospital, Dooradoyle, Co Limerick, Ireland; nialldavis2001@yahoo.com 